Please do not remove this page

RMIT

UNIVERSITY

\title{
Can artificial life emerge in a network of interacting agents
}

Murthy, Venu; Krishnamurthy, E

https://researchrepository.rmit.edu.au/esploro/outputs/9921862506801341/filesAndLinks?institution=61RMIT_INST\&index=null

Murthy, V., \& Krishnamurthy, E. (2006). Can artificial life emerge in a network of interacting agents. 2006 IEEE Proceedings - International Conference on Fuzzy Systems, 106-113.

https://doi.org/10.1109/FUZZY.2006.1681702

Published Version: https://doi.org/10.1109/FUZZY.2006.1681702

Repository homepage: https://researchrepository.rmit.edu.au

(C) 2006 IEEE

Downloaded On 2023/04/26 21:45:21 +1000

Please do not remove this page 


\title{
Can Artificial Life Emerge in a Network of Interacting Agents?
}

\author{
V.K.Murthy ${ }^{1}$, E.V.Krishnamurthy ${ }^{2}$ \\ ${ }^{1}$ School of Business Technology, R.M.I.T., Melbourne, Victoria 3000, Australia \\ kris.murthy@rmit.edu.au \\ ${ }^{2}$ Computer Sciences Laboratory, The Australian National University, Canberra, ACT 0200, Australia. \\ abk@mail.rsise.anu.edu.au
}

\begin{abstract}
An interacting multi- agent system in a network can behave like a nature - inspired Smart System (SS) exhibiting the four salient properties of an Artificial Life System (ALS): (i) Collective, coordinated and efficient (ii) Self -organization and emergence (iii) Power law scaling or scale invariance under emergence (iv) Adaptive, fault tolerant and resilient against damage. We explain how these basic properties can arise among agents through random enabling, inhibiting, preferential attachment and growth of a multiagent system. However, the quantitative understanding of a Smart system with an arbitrary interactive topology is extremely difficult. Hence we cannot design a general purpose programmable Smart system. However, for specific applications and a predefined static interactive topology among the agents, the quantitative parameters can be obtatined through simulation to build a specific SS.
\end{abstract}

\section{INTRODUCTION}

The Science of Smart systems (SS) is of recent origin. It is a highly interdisciplinary area and the definitions have not yet been well-formulated and vary between fields although the basic properties of these system are nature-inspired.In Physics, SS are ensembles whose dynamics can be monitored or modified by distributed sensors and actuators, in accordance with an integrated control law to accommodate time-varying exogenous inputs or changing environmental conditions. The SS exchanges energy, entropy and matter with the environment. On an average SS exports entropy, it operates far from equilibrium, beyond a critical distance from the equilibrium state, positive and negative feedback are present and the system is nonlinear.

In engineering, an SS is one that monitors itself and /or its environment in order to respond to changes in its conditions. This can result in (i) Adaptation (ii) Self-repair and (iii) Optimisation of resources.

Biosystems characterize SS by the properties:

1.The processing of information is distributed and is monitored

2.The communication system is reconfigurable .

3.The system is highly adaptive and acquires knowledge

through interaction with its environment.

4.The energy consumption is optimized .
5.The system is self -organizing; it exhibits emergent properties which are not predictable, in advance from the properties of the individual local interactions among its components and the environment.

Here, we define SS with the following four properties that are reflected in each of the above viewpoints.

1. Interactive, Collective, and efficient

An SS consists of a large number of components and interacting with the environment. They collectively and cooperatively perform actions, coordinating their actions if there is competition, to obtain maximal efficiency.

2. Self- organization and emergence

The total dynamic behaviour arising due to cooperation between different parts of the SS can lead to a coherent behaviour of the entire system that can change by a large amount when the values of a control parameter changes by a small amount (sensitivity). These changes have similarities to the phase transition encountered in physics, where local changes result in a global change in which new properties emerge abruptly. These new properties of the SS are not predictable, in advance from the properties of the individual interactions. In particular, under emergence, the many degrees of freedom arising due to its component parts collapse into a fewer new ones with a smaller number of globally relevant parameters. This means the properties of self -organizaion cannot be predetermined and the system evolves on a qualitatively new level with respect to its global constraints.

3. Power-Law Scaling or Scale-Invariance Property

Power -law scaling relation arises for the emergent properties of the SS. Dorogovtsev and Mendes [7] prove that this property is essential for fault resilience and selforganization.

4. Adaptive, fault-tolerant and Resilient against damage

SS are always flexible to change-they can self-modify their past behaviour and adapt to environmental changes, available resources, as well as, tolerating failures or noncooperation of some of their components. We can include mutation and reproduction in this property.

It appears that these four properties are interrelated to selforganization, stigmergy and self-assembly, as well as, to 
systems exhibiting positive metric entropy, self-similarity and the small world phenomena. It is hard to separate these concerns as they form fuzzy boundaries [20].

In this paper we consider the following issues:

1.What kind of models are needed for studying SS?

We discuss three kinds of models that have a direct bearing on these issues.:(i) Fractal and percolation model, (ii) Chaotic and nonlinear dynamical model and (iii) Topological (network) or graph model.

2.Is the emergence of SS analogous to the critical phenomenon in physics or percolation? What are the suitable parameters to describe this phenomenon?

We discuss this aspect from the point of view of geometric parameters such as: Lyapunov exponents, strange attractors , metric entropy, as well as, topological indices such as, Cluster coefficient, Average degree distribution and the correlation length of the interacting network.

3.Can we design a general purpose programmable smart system?

The answer is no, if we want to have an SS with exactly specified propeties.However, special purpose Smart systems with approximate statistical properties can be designed by using simulation procedures and parameter tuning.

Section II describes the fractal and percolation models. Section III explains the role of positive metric entropy condition in SS. Section IV outlines the statistical properties of disordered networks and their role in SS. Section V deals contains the simulation aspects of SS; these are illustrated by a few examples-ant colony heuristic based on group swarming, human-animal trails and stigmergy. Section VI briefly considers the engineering of SS; this is analogous to the synthesis of heterogeneous materials from a knowledge of the micro-structural information [26]. Section VII outlines the negative results arising due to the statistical nature of the models available and our inability to compute the exact parameters numerically.

\section{FRACTAL AND PERCOLATION MODELS}

An SS has an associated data domain or space. In nature, this space is usually the three dimensional space; it is called the geometric dimension of the SS. When the system is placed in an environment, it communicates through its surface area. Since the amount of communication is proportional to the surface area, a simple way to control the communication rate is to choose a suitable geometrical or topological structure that can spontaneously and easily be modified to vary the surface area, Bunde and Havlin [4], Grimmett [10], Hilborn [11], Moon [16], Torquato [26], Wolfram [28]. In biology,chemistry, and materials science, where surface phenomena play a crucial role, nature has preferred the evolution of fractal objects. Such a choice can optimize the interaction with the environment and provides for adaptation and the survival. In heterogeneous chemistry, the structure and geometry of the environment at which the reaction takes place plays an important role. It alone can dictate whether a reaction will take place at all. The geometric parameter is the fractal dimension. In fact environmental interaction can change geometrical features and conversely, geometrical feature modify the interaction; see Chapter on "Fractal Analysis" by Avnir et al. in Bunde and Havlin [4],Torquato[26].

To engineer a nature -inspired Smart System using computing agents, therefore, the agents should be able to alter the pattern of their communication pathways, namely, the topology and geometry at will, resulting in the change of a fractal dimension.Examples of such systems abound in nature: lungs, flock of birds,ant-swarms, animal- human trails, Smart materials [23],[26].

However, we still need yet another model that can provide us a tool based on probabilities to compute the connectivity structure among the components in a network arising from interactions. This model is the Percolation model, Grimmett [10], Torquato [26]. The percolation theory is concerned with the study of the diffusion / penetration of certain materials from an environment into the material of system placed in that environment through the boundary or surface of the system. It has been found experimentally that such a penetration is determined by a single parameter $\mathrm{p}$. We call a particular value $p=p(c)$ the "threshold" for percolation, if the pathways are infinite when $p>p(c)$ and the pathways are nonexistent or have limited access within the material of the system when $p<p(c)$. The value $p(c)$ is called the critical point. Also we say that there is a percolation above $p(c)$. The region above $p(c)$ is called the supercritical phase and the region below $p(c)$ is called the subcritical phase. Also when $p=p(c)$ the infinite open cluster or pathway is called an incipient infinite cluster.

Percolation theory is studied using an internal connected structure of the material of the system, either as a lattice or as a connected graph. Also it is studied as a "bond percolation" in which we study the percolation through the edges of the lattice or a graph; or as a "site percolation" in which we study the percolation through the lattice points or vertices of the graph or as a "mixed percolation" involving edges and vertices.

For example, consider a square lattice, where each site is occupied randomly with probability $\mathrm{p}$ or empty with probability (1-p). At low concentration $p$ the occupied states are either isolated or forms small clusters. Two occupied sites belong to the same cluster if a path of nearest neighbour occupied sites connects them. When $\mathrm{p}$ is increased, the average size of clusters increases. At a critical concentration $\mathrm{p}(\mathrm{c})$ a large cluster appears which connects the opposite edges of the lattice. This cluster is called the infinite cluster since it diverges when the size of the lattice is increased to infinity. When $p$ increases further the density of the cluster increases, since more and more sites become part of the infinite cluster, and the average size of the finite clusters decreases. The value of the percolation threshold $\mathrm{p}(\mathrm{c})$ depends explicitly on the type of lattice. In site percolation $p(c)=0.593$ for square lattice. This means, if a square of unit area is filled in with agents whose range of influence is in a square lattice, then it is adequate that about $60 \%$ of the lattice is filled so that there will be a percolation path between the edges of the square .e.g., if there are 100 lattice points it is adequate to cover at least the $60 \%$ of the area of 
the square or 60 communication agents are used where each agent can communicate with its eight neighbours, diagonals and adjacent squares. The fractal dimension $\mathrm{d}(\mathrm{f})$ of the percolation of the square lattice of dimension 2 is $d(f)=$ $91 / 48=1.9$.

The density of the agents $<\mathrm{n}>=$ Number of agents/ lattice size vs Clustering obtained through simulation. In this simulation, we start with a square lattice of side length $\mathrm{L}$ and some numbr $\mathrm{p}, 0 \leq \mathrm{p} \leq 1$; we generate a random number in $[0,1]$ for each lattice cell and say a cell is empty if the random number is less than $\mathrm{p}$. The patterns can be generated for various values of $p$. Denoting by $M(L)$ the number of cells in the larest cluster, M(L) scales as:(i) $\ln (\mathrm{L})$ for $\mathrm{p}<\mathrm{p}(\mathrm{c})$; (ii) $\mathrm{L}^{\mathrm{d}}$ for $\mathrm{p}=\mathrm{p}(\mathrm{c})$ and (iii) $\mathrm{L}^{2}$ for $\mathrm{p}>\mathrm{p}$ (c) where $\mathrm{d}=1.9$ and power-law scaling holds at the critical threshold.

Random fractals can contain disjoint clusters(or multiply connected).Their topological structure can change dramatically, when a continuously varying parameter increases through a critical value resulting in a simply connected path, [10], [26]. Thus unforeseen new paths can emerge between points of interest beyond a critical threshold, e.g., nest to the food when communication takes place among agents in an environment.

Thus the fractal and percolation models play key roles in engineering a nature -inspired Smart Systems, Grimmett [10], Torquarto [26],Watts [27], Barabasi et al [1]. However,the dynamics of the resulting SS is in general, highly nonlinear implying that it is not possible to obtain analytical expressions for its behaviour.We need Monte Carlo simulation to compute the percolation threshold and related parameters [26].

\section{POSITIVE METRIC ENTROPY CONDITION}

The properties of SS mentioned in Section I and the requirement mentioned in Section II having a readily modifiable connectivity pattern among its components with a fractal structure, belong to those of both the computational and the nonlinear dynamical systems [11], [14]. The fractal communication structure is very sensitive to small parameter changes and can lead to an abrupt change in behaviour, e.g bifurcation. Fractal dimension, metric entropy, and Lyapunov exponent serve as important invariants to quantify the behaviour of such a non-linear system, Falcioni et al. [9]. A system with negative Lyapunov exponents imply stability and a system with the positive Lyapunov exponents imply instability and the presence of positive feedback. The positive Lyapunov exponent in a nonlinear system implies a positive metric entropy; it plays the same role as entropy export in thermodynamical system leading to nonequilibrium states. Such a system is no longer expressible as a sum of its component parts and the system functions as a whole leading to what is known as "Emergence". In such a case, the algorithmic independence among the subsystems is lost. In general, the evolutionary rules of systems with zero and negative entropy are predictable ( or Lyapunov exponent less than or equal to zero) and such rules are non-algorithmic, or not exactly predictable for positive entropy machines.
Well- structured objects - such as: Context free grammars, regular grammars and serial-parallel graphs, regular graphs, lattice graphs that are generated using deterministic algorithms provide for easy description through functional rules, and hence have zero metric entropy. Such systems with non-positive metric entropy are "Turing or algorithmically expressible" with a finite set of evolutionary rules. Systems with a positive metric entropy represent "disordered" machines and their evolutionary rules are in general, "Turing non-expressible". The metric entropy can distinguish the two major classes of machines: ordered $(\mathrm{O})$ and disordered) (D) machines

1.Ordered or Zero Metric Entropy Machines (O)

These are Completely structured, Deterministic, Exact behaviour (or Algorithmic) Machines. This class contains, the machines in Chomskian hierarchy:

(i) Finite state machines ( regular grammar),

(ii)Push-down stack machine (context-free grammar),

(iii)Linear bounded automata (context sensitive grammar)

(iv) Turing machines that halt

(v) Exactly integrable Hamiltonian flow machines.

These machines are in principle, information loss-less and instruction obeying; their outputs contain all the required information, as dictated by the programs.

\section{Positive Metric Entropy or Disordered Machines (D)}

Nonintegrable positive entropy machines exhibit various degrees of irregular dynamics [9]:

(i) Ergodicity: Here the set of points in phase space behave in such a way that the time-average along a trajectory equals the ensemble average over the phase space. The term "Ergodicity" means statistical homogeneity; here, the trajectory starting from any initial state can access all other states in the phase space.

(ii) Mixing: The initial points in the phase space can spread out to cover it in time but at a rate weaker than the exponential (e.g. inverse power of time).

(iii) Bernoullicity, K-flow or Chaos: The trajectories cover the phase space in such a way that the initially neighbouring points separate exponentially and the correlation between two initially neighbouring points decays with time exponentially. It is with this class of irregular motion we define classical chaos. These lie in the border and beyond the Turing computable region;that is they belong to partial recursive schemes leading to undecidability.

(iv) Non-equilibrium systems: Systems exhibiting emergent behaviour - such as Chemical and Biological machines and living systems, [19],[26],[30].

Each of the above properties imply the all the preceding properties, e.g.,within a chaotic region the trajectories are ergodic on the attractor and wander around the desired periodic orbit.

The properties of an SS is governed by three important parameters: Metric Entropy, Lyapunov exponents, and Fractal dimension. There is a close realationship between positive Lypunov exponents and fractal dimensions.

If $\mathrm{h}(1), \mathrm{h}(2), \ldots, \mathrm{h}(\mathrm{j})$ are Lyapunov exponents in ascending order that are greater than zero, and $, \mathrm{h}(\mathrm{j}+1), . . \mathrm{h}(\mathrm{k})$ are less than zero in descending order, then we have $\mathrm{j}$ stretching and 
( $\mathrm{k}-\mathrm{j})$ contracting directions in the $\mathrm{k}$ dimensional trajectories, then the quantity, Lyapunov dimension, Moon [16]:

$d(L)=j+1 / h(j+1) \sum_{i=1}^{j} h(i)$. It is conjectured that

$\mathrm{d}(\mathrm{L}) \leq \mathrm{d}(\mathrm{b})$ the box-counting dimension.

Hence, to understand an SS quantitatively, we need to know the spatial structure of attractors and the temporal aspects of the trajectories. The former provides information about phase transitions, while the latter tells us whether a trajectory governed by a positive Lyapunov exponent falls in a given attractor. Real systems including biological systems are nonstationary in which the parameters are changing with time. Thus quantitative understanding of a Smart syatem is extremely difficult except through simulation. To model SS, we need to combine zero and positive entropy machines; that is we introduce positive entropy through the chaoticity (deterministic randomness) or stochasticity (statistical randomness) in appropriate decision or control steps of a deterministic machine.

The positive entropy endows SS with several powerful features:

(i) It provides ergodicity of search orbits. This means every point in the set of accessible states is approached arbitrarily closely during the iteration. This property ensures that searching is done through all possible states of the solution space since there is a finite probability that an individual can reach any point in problem space with one jump.

(ii) It provides solution discovery capabilities (as in genetic programming-GP) due to embedded randomness.This property arises due to the fact that chaotic orbits are dense and has positive Lyapunov exponent and two initially close orbits can separate exponentially from each other with a possibility of falling into a desired attractor.

(iii) It provides a common linking thread among several disciplines; e.g., Genetics(genetic-algorithms), Diffusion, Thermodynamics (simulated-annealing), Statistical Mechanics ( Particle transport); Complex Systems (Active walker, Self- organization and percolation models).

In distributed computing with agents, and amorphous computing, a very large disordered interconnection network having a positive metric entropy arises in the state space. Such networks have various statistical and nonlinear dynamical properties- random, small-world and scale free networks- having different fractal dimensions and emergent properties, Watts [26], Strogatz [25], Dorogovtsev [7], Barabasi et al., [1], Ben Naim et al [2], Newman [17], Zaslavsky [31].

To realize self-organization, therefore, the agents need to strategically control their links with other agents based upon some policy. Such policies are Turing non-expressible and are entirely empirical and statistical.

\section{CLASS OF DISORDERED NETWORKS}

We now classify the connection patterns arising among interacting agents, since they play a key role for applications to various problems. We start with a graph model as the basis for communication networks among the agents. Three important statistical properties of the networks based on the neighbourhood of nodes in a graph are used to classify the disordered networks: (i) Random networks (ii) Power-law scaling networks, and (iii) Small World Networks. For an excellent survey of complex networks, see, Newman [17].

Let us consider a finite graph $\mathrm{G}(\mathrm{V}, \mathrm{E})$ where $\mathrm{V}$ is the set of $\mathrm{n}$ nodes and $\mathrm{E}$ the set of edges. Let us assume that the graph is represented as an adjacency matrix $A$ with $A(i, j)=1$, if there is an edge from node $i$ to node $j$; and $A(i, j)=0$, otherwise.We assume $\mathrm{A}(\mathrm{i}, \mathrm{i})=0$, that is no self loops. The following local and global parameters are derived from adjacency matrix:

Average degree: $\mathrm{K}=1 / \mathrm{n} \sum_{\mathrm{i}} \mathrm{k}(\mathrm{i})$, and $\mathrm{k}(\mathrm{i})=\sum_{\mathrm{j}=1}^{\mathrm{n}} \mathrm{A}(\mathrm{i}, \mathrm{j})$, or

$\mathrm{k}(\mathrm{i})$ is the degree of node, $0 \leq \mathrm{K} \leq(\mathrm{n}-1)$

The Characteristic path length $L$ measures the global property,namely, the average path length of the network, defined by:

$\mathrm{L}=2 / \mathrm{n}(\mathrm{n}-1) \sum_{\mathrm{i}=1}^{\mathrm{n}-1} \sum_{\mathrm{j}=\mathrm{i}+1}^{\mathrm{n}} \mathrm{L}(\mathrm{i}, \mathrm{j}) ; 1 \leq \mathrm{L} \leq(\mathrm{n}-1)$

The cluster coefficient $\mathrm{C}$ is the average of C(i), where C(i) is defined by:

$\mathrm{C}(\mathrm{i})=2 \sum_{\mathrm{j}=1}^{\mathrm{n}} \sum_{\mathrm{k}=1}^{\mathrm{n}} \mathrm{A}(\mathrm{i}, \mathrm{j}) \mathrm{A}(\mathrm{i}, \mathrm{k}) \mathrm{A}(\mathrm{k}, \mathrm{j}) /[\mathrm{k}(\mathrm{i}) \mathrm{k}(\mathrm{i})-1]=$

Number of E(i) existing between $\mathrm{k}(\mathrm{i})$ neighbours of node $\mathrm{i}$ /Total number of possible edges $[\mathrm{k}(\mathrm{i})(\mathrm{k}(\mathrm{i})-1] / 2$.

or, $\mathrm{C}=1 / \mathrm{n} \sum_{\mathrm{i}} \mathrm{C}(\mathrm{i})$. Note that $0 \leq \mathrm{C} \leq 1$

(i) Random Network:

In Random network the degree distribution is a binomial or Poisson distribution in the limit of a large graph. Most of the nodes have the average degree and few nodes have more than average and few nodes have less than the average degree. Also $\mathrm{L}$ and $\mathrm{C}$ are small in random graphs.

(ii) Scale-free Network:

In this network, many nodes have a few links and a few nodes have many links. The distribution of the degree of the nodes has an unusual fat-tailed form or a power-law scaling property.; namely the $\mathrm{P}(\mathrm{k})$ the degree distribution of a network is given by: $\mathrm{P}(\mathrm{k})=\mathrm{k}^{-\mathrm{g}}$. where $2<\mathrm{g}<3$.

This power-law degree distribution or scale-invariant property arises from two kinds of operations on a random graph, Barabasi, Albert and Jeong [1].

1. Creating new nodes: Growth of the graph by adding new nodes into an initial group of nodes as time progresses and 2. Preferential attachment of Links: The new nodes created are linked to old nodes, with a probability based on certain dominant properties the old nodes possess, e.g. the nodes having a higher degree (or attractiveness), chemical or physical interaction strength. In each case, the neighbourhood is appropriately defined as a conceptual graph. As the network grows the ratio of well-connected nodes to the number of nodes in the rest of the network remains nearly a constant.Dorogovtsev et al. [7] prove that 
the range $2<\mathrm{g}<3$ is crucial to have the following properties, see also [1].

(a) Self-organization and

(b) Resilience against random damage.

(iii)Small-world graphs:

A graph is called a small -world graph, by Watts [27], if it exhibits the two properties given below when compared to a random graph of same number of nodes and average degree:

1. Higher clustering coefficient $C$ closer to unity and

2. Smaller Correlation length $L$.

L scales logarithmically with the number of nodes.This measures a global property. It results in a compact networkwhere the average length of the shortest directed path between two randomly chosen nodes is of the order of the logarithm of the size of the graph. This property ensures that communication time between distant neighbours is not exponential in complexity and interaction can take place over long ranges, yet preserving causality. This is called the small world effect and the short path is also called a "wormhole". Therefore, in agent based systems, where a very large number agents are interconnected, the small-world network, can permit distant neighbours to interact with an efficient flow of information.

\section{SIMULATING SELF- ORGANIZATION}

An agent is a system that is capable of perceiving events in its environment or representing information about the current state of affairs and of acting in its environment guided by perceptions and stored information [29]. Here, we consider the evolution of a massively many (a multiset of) agents into an SS in a network. That is we are interested in connected pathways among agents satisfying certain special properties, such as-minimal cost route, clustering of agents, resilience of conectivity among the agents under failure, attractors, phase transitions and so on. These properties depend upon the temporal dynamics of the system as embodied in the Lyapunov exponents and the spatial structure of the dynamical system's attractors.

Multi-set Agent-based simulation [14] or realization, starts with simple rules of interaction among the individual agents that drive the system to the complex behaviour observed. It consists of the following features:

(i) The multiset-agent system is made up of several single agents [29] whose information is structured in an appropriate way to suit the problem at hand.

(ii) A set of local interaction rules that prescribes the context for the applicability of the rules to the agents. Each rule consists of a left-hand side (a pattern or property or attribute) describing the conditions under which the agents can communicate and interact, and a right hand side describes the actions to be performed by the agents, if the rule becomes applicable based on deterministic or probabilistic criteria.

(iii) A control strategy that specifies the manner in which the agents will be chosen and interaction rules will be applied, the kinetics of the rule- interference (inhibition, activation, diffusion, chemotaxis) and a way of resolving conflicts that may arise when several rules match at once. (iv) A coordinating agent or an agent by itself selfevaluates the performance of each agent to determine the effectiveness

(absolute or shared fitness) of rule application and the resulting action.

(v) Interaction -Based: The computations are interpreted as the outcome of interacting agents to produce new agents (reproduction), or agents with modified attributes (mutation), according to specific rules. Hence the intrinsic (genotype) and acquired properties due to interaction (phenotype) can both be incorporated in the agent space. Since the interaction rules are inherently parallel, any number of actions can be performed cooperatively or competitively among the subsets of agents, so that the new agents evolve towards an equilibrium or a self -organizing state.

(vi) Content-based activation of rules: The next set of rules to be invoked is determined solely by the contents or properties of the agent-space, as in the context of chemical reactions or environmental interactions.

(vii) Each agent may retain its current belief or revise its belief as a result of receiving a new message by performing a local computation. If it revises its belief, it communicates its revised state of belief to other concerned agents; else it does not revise its solution and remains silent..

We assume that there are two ways in which mutual interaction can take place between any two agents:

1.Enabling dependence (ED): Agents $\mathrm{A}(\mathrm{i})$ and $\mathrm{A}(\mathrm{j})$ are called enable dependent (or dataflow dependent) through $\mathrm{A}(\mathrm{k})$ if the messages from $A(i)$ creates the required precondition in $\mathrm{A}(\mathrm{k})$ and results in a message to $A(j)$ and creates the required precondition in $\mathrm{A}(\mathrm{j})$ to act (fire).

2.Inhibit dependence (ID): Agents A (i) and A (j) are called inhibit dependent, if the actions of $A$ (i) do not create the required precondition in $A(k)$ needed by $A(j)$ and prevents it from executing any action.

In order that a multiset of agents behaves like an SS, it should exhibit the four properties mentioned earlier. This will require that the agents are not centrally controlled, but can either enable (link) or inhibit (de-link) with other agents on their own,based on the information available from their environment.This will correspond to adaptation. This requires that the agents have adequate memory, intelligence,and pre-knowledge of the network of other nodes in the network.

We can use two different approaches inspired by Nature to enable (connect) or inhibit (disconnect) agents to form an interactive network:

1.Use of markings similar to a chemical gradient or diffusion mechanism or a communication field (Agents having simple intelligence, e.g ants, physical particles):

This provides a common spatial resource, where each agent can leave a mark that can be perceived by other agents. In essence, the agents use the space to construct a geometrical or a topological pathway that is preferentially traversed by many agents, resulting in a power-law scaling or small world beyond a critical threshold. From a practical viewpoint, in the agent model, the shared landscape can be simulated through a blackboard and self-avoiding walks can be 
simulated using a table that stores the locations visited earlier.

2.Use of a positive feed-back or nonlinear response to the information available from knowledge sources, other agents may possess by connecting or disconnecting with other agents at random:

This would require that each agent knows what other agent knows, and how much they know measured in a taxonomic scale (Agents with more complex intelligence) so that each agent can have a score about its neighbours to link, delink and form clusters. This would result in nonlinear iterative schemes among the agents. Here, individual agents are points in space, and change over time is represented as movement of points, representing particles with different properties and the system dynamics is formulated using the rules:

\section{(1) Stepping (or local coupling) rule:}

The state of each individual agent is updated or revised in many dimensions, in parallel, so that the new state reflects each agent's previous best success.

\section{(2) Landscaping (or global coupling) rule:}

Each agent assumes a new best value of its state that depends on its past best value and a suitable function of the best values of its interacting neighbours, with a suitably defined neighbourhood topology and geometry.

All agents in the universe or selected chunks are updated using rules (1) and (2).

The above two rules permit us to model Markovian random walks which is independent of the past history of the walk and non-Markovian random walks, dependent upon past history- such as self-avoiding, self-repelling and active random-walker models. This can result in various kinds of attractors having fractal dimensions presenting a swarm-like, flock-like appearances depending upon the Jacobian of the mapping, Wolfram [28].

We now describe a multiset of agents based selforganizing heuristic that works along with inferences, beliefs and computation. We assume that the number of agents are very large to exhibit the statistical features. The agents use randomization in the application of the rules and interconnectivity among them that can alter the important neighbourhood properties; this results in different kinds of disordered networks with fractal dimensions.

\section{A. Swarm of Agents and self-organization}

The multiset of agents paradigm [14] to realise particle swarms [12] has many advantages.

(i) The fundamental principle in swarm intelligence is cooperation and knowledge sharing. The multiset of agents approach can realise both exploration and exploitation by grouping the particles in different zones of the search space to set up cooperation and competition among members of same group and different groups. Using different grouping prevents possible quasi-ergodic behaviour that can arise resulting in the entrapment of the orbit in isolated regions, since the grouping amounts to using several Markov chains, with the initial states reasonably apart, in the search space. (ii) Different types of interaction topologies (such as the wheel, the ring, the star and other lattice structures [12], can be assigned within and among different groups and their influence can be studied .

(iii) If we need to optimize several objectives the multiset of agents swarming can be useful .

(iv) Since there are different groups, evolutionary paradigm approach can be tried, by annihilating unfit populations and creating new populations.

The Ant Heuristics [2],[3],[6],[12] are based on the model of real ants finding optimal solutions to the shortest path between their nests and the food sources. Each ant leaves a trail of pheromone, thereby creating a new landscape so that other ants are attracted towards the food source by the scent. This problem is analogous to modifying a random graph that grows with time with preferential attachment to those strong pheromonal nodes leading to the food source from the nest. The optimal path turns out to be the path with a maximum density of the scent allowing for evaporation of the scent with time and degradation of the scent with distance. The communication among the ants take place through a shared landscape that establishes a link with the other agent nodes with the same belief and forming clusters.

To start with, the agents initialize their beliefs, by randomly linking for information.Then with time they update their beliefs through preferential linking to nodes with maximal pheromone intensity and forming local (groups) clusters. Then they modify the landscape further, by contacting other clusters to obtain collective intelligence, and reach an equilibrium state resulting in an optimal path from the nest to the food source.

The above search heuristic was simulated with a multiset of agents consisting of 100 groups each with 500 agents and the performance studied. In comparison to using a single group of 50000 agents, the multi-group performance was markedly better. Using multi-swarms rather than a single swarm and setting up competition within a swarm and between different swarms results in a more efficient heuristic in which preferential attachment happens to high degree nodes, leading to "scale free distribution". In this set-up ; if some of the individual nodes or ants are randomly destroyed, the heuristic is not affected at all exhibiting the resilience of the scale-free distribution under random failure. However, if some of the hubs of well-connected group of nodes are selectively destroyed, the heuristic fails.

Also in this heuristic, since the initial tendency is to form local links, this results in higher clustering and if many nodes find the same nodes to link with no degree restriction, we obtain a correlation length smaller than the random network.

Also (i) if some agents are assumed to be aging, noncoopertaive and they are allowed to die, and (ii) we limit the number of linkings to each agent by a cost factor, then the scale-free distribution is markedly affected. Our estimate of the box-counting fractal dimension of the swarm lies between 1.7 and 1.9 (resembling Diffusion limited aggregation [26] ). This value is lower than its expected value 2 ; it may be due to the approximate procedure we used 
to convert swarm trajectory into a planar lattice pattern, to estimate the power law exponent.

Simulation results show that the swarm network topology is very sensitive to the nature of interaction and threshold values, cost and aging of nodes. This emphasises the fact that the algorithmic structure has broken down and the system is self-organizing.

\section{B Simulating Animal-Human Trails}

Active walker models in which animals or humans interact through indirect communication mediated by the environment and leave a purposeful trail either from nest to the food source or between other points of interest, can be interpreted qualitatively as a small world phenomena on graphs, or as percolation phenomena in lattices, by choosing a proper lattice structure (square or hexagonal) tiling the space. These trails result due to agglomeration process of several walkers moving arbitrarily leaving markings (clearing vegetation or leaving chemicals), but eventually producing an attractive effect on another walkers. Clearly, there is a preferential choice among the possible trails and the most frequently used trails combine to become popular. Also rarely used old trails disappear and frequently used trails are reinforced although many new entry points may arise and destinations may branch off. Also fitness is evaluated for each trail as to its cost and utility [8], [21].

\section{Genetic Programming approach to Graph Growth}

The problem of growing a graph until it reaches selforganized criticality through interaction is closely related to Genetic Programming (GP), Koza [13]. In GP each program construct is a tree constructed from tasks, functions and terminal symbols. Then we perform crossover and mutation by swapping program sub-trees leading to feasible programs, taking care of the nature and type of the task. These operations resemble Metropolis-Hastings-Monte-Carlo methods , [17], [24] to create transitivity in a graph from a given node to a desired attractor node.The GP operations correspond to an ergodic move-set in the space of graphs with a given set of parameters and repeatedly generating the moves and accepting them with probability $\mathrm{p}$ or rejecting them with probability (1-p)

Suitable move-sets are: creation of new nodes, aging and annihilation of nodes, Mutation -movement of edges from one place to another, mating -swapping edges of the form $(\mathrm{s}, \mathrm{t}),(\mathrm{u}, \mathrm{v})$ to $(\mathrm{s}, \mathrm{u}),(\mathrm{t}, \mathrm{v})$, adding new edges based on a cost function. Such moves can create a phase transition (or percolation) to reach a global goal through successive local goals. An important aspect in GP is the fitness of the individual program generated locally and globally.In selforganization, ideally, one requires that the fitness is a selfawareness function i.e. the individual who does the work evaluates itself, ensuring that the global fitness is guaranteed. This is widely prevalent in Nature for activities such as: nest building (stigmergy), food searching (foraging).

\section{Simulating Stigmergy}

In Stigmergy (a term coined by French biologist Pierre-Paul Grasseis), two organisms interact indirectly through the environment. When one of them modifies the environment, the other responds to the new environment at a later time, e.g., nest building in insect societies. Starting with a basic pattern the nest grows by adding new material. The resulting structure produces different types of stimulus and responses from other members to build the nest further. This has all the features of a co- evolutionary algorithm, Koza [13] in the sense it is a mutually bootstrapping process, that is driven by relative fitness (not absolute fitness). (i) We have a population of competing and cooperating individuals who exchange local information and interact. (ii)The shared fitness of the individuals piece of contribution is evaluated. (iii)Fitness based selection of sites for building the walls of a nest. (iv)Probabilistic variation of the nest based on builders.

The Wasp nests,Camazine et al.[5], are built by preferential attachment of a new part with the old assembly with probabilities $0.55,0.06,0.39$ respectively to fill in 3 walled hexagon, 2 walled hexagon, and 1 walled hexagon. That is, the sites are rank ordered and the local selection principle and preferential attachment ensures local fitness and promises global fitness and results in a scale invariant property for the shape. This whole process of nest building resembles a competitive game with a limited local information. The local fitness ensures global fitness because of the geometrical scale invariance.

In wasp nests, the walls are corrugated to achieve selfsimilarity of the whole structure, since hexagons cannot tile the plane. Further, wasps use the preferential attachment rule that achieves both the self-similarity of the structure and geometrical compactness to have the largest possible area (volume) with the least perimeter (surface area).

\section{E. Synchronization among agent population}

A simplest adaptive system arises in "Synchronization". Here two oscillatory systems (or repetitive systems) adjust their behaviours relative to each other so as to attain a state where they work in unison. This is a universal phenomenon. When two nonlinear oscillators interact, mode locking may occur whenever a harmonic frequency of one mode is close to a harmonic of other. As a result nonlinear oscillators tend to lock to one another so that one system completes precisely $\mathrm{p}$ cycles each time when another system completes q cycles ( $p, q$ integers). Agents provided with nonlinear oscillatory capabilities can couple through different choice of interacting functions. This kind of coupling can result in the emergence of dynamical order and the formation of synchronous clusters and swarming. Such SS can function like Chemical systems, Biological systems, Molecular machines and will have applications in designing task specific biomorphic robotics [15], [18], [25].

\section{ENGINEERING SS}

Since an SS is inherently nonlinear and hysteretic, agents made out of Piezoceramics, magnetostrictives, ShapeMemory Alloys (SMA), Electrostrictive polymers, Ferromagnetic SMA, can serve as components for specific 
applications, [23],[26]. However, the central question in designing SS is how to program the components so that the system as a whole self organizes. This is the basic question addressed in the design of Amorphous computers and Spray computers, as well as in the synthesis of heterogeneous materials with certain specified macro properties from a knowledge of the properties of the microsturctures, Torquato [26]. Emergence is a global behaviour (or a goal) that evolves from the local behaviours (goals) of components, Serugendo [22]. The evolutionary rules for arriving at the global goal is non-computable, since it cannot be expressed as a finite composition of computable deterministic function of local goals for any arbitrary problem domain. Thus we cannot design a general purpose programmable Smart system if we want to have an SS with exactly specified propeties.

However, for specific applications and a pre-defined interactive topology among the agents, the geometric, dynamical, topological parameters, and statistical properties can be obtained through simulation and tuned to build a specific SS.

\section{CONCLUSION}

We described some important properties a smart system (SS) need to possess and the models needed to understand SS. Multi- agent systems in a network can exhibit the properties of both the computational and dynamical systems and can undergo phase transition and emerge into an SS exhibiting the salient features of Artificial Life systems (ALS). However, to understand SS quantitatively, we need to obtain the spatial structure of attractors and the temporal aspects of the trajectories, and topological or graph parameters. The first two aspects provide information about phase transitions and tells us whether a trajectory governed by a positive Lyapunov exponent falls in a given attractor. The topological aspects give us a statistical behaviour of the network of connectivity among the agents. Real systems including biological systems are nonstationary, in which the parameters are changing with time. Thus quantitative understanding of a Smart system using the above parameters with arbitrary time varying interactive topology seems not possible. These negative results arise due to the statistical nature of the models available and inability to compute the exact parameters numerically. However, for specific applications and a pre-defined static interactive topology among the agents, the statistical parameters can be obtained through simulation and tuned to build a special purpose SS.

\section{REFERENCES}

[1] A.Barabasi and R. Albert, " Emergence of scaling in random networks", Science, vol.286,pp.509 - 512,1999.

[2] E. Ben-Naim et al.,(Eds)., Complex Networks, Lecture Notes in Physics, vol.650, Springer Verlag, New York 2004.

[3] E.Bonabeau, et al..Swarm Intelligence, Oxford University Press, London, 1999.

[4] A.Bunde and S. Havlin, Fractals in Science, Springer Verlag, New York.1994.

[5] S.Camazine,Self-Organization in Biological Systems, Princeton University Press, Princeton,2002.

[6] M.Dorigo et al,.Ant Algorithms, Lecture Notes in Computer Science, vol.2463, Springer Verlag, New York, 2002.

[7] S.N.Dorogovtsev and J.F.F.Mendes, Evolution of Networks, Oxford University Press, Oxford ,2003.

[8] W.Ebeling andF.Schweitzer, "Self-organization, Active Brownian dynamics and biological Applications", Nova Acta Leopoldina, vol.88, No.332,2003,pp.169-188

[9] M.Falcioni et al, “Kolmogorov's legacy about entropy, Chaos and Complexity", Lecture Notes in Physics, vol.636, Springer Verlag, New York , 2003, pp. 85-108

[10] G. Grimmett,Percolation, Springer, New York,1999.

[11] R.C.Hilborn, Chaos and Nonlinear Dynamics, Oxford University Press, Oxford, 2003

[12] J.Kennedy, "Swarm Intelligence", in Handbook of NatureInspired\& Innovative Computing, Ed: A.Zomaya, Springer Verlag, New York, 2006, pp.187-221.

[13] J.R.Koza,Genetic programmingIII, Morgan Kaufmann, San Francisco, 1999.

[14\} E.V. Krishnamurthy et al, "Multiset Rule-based Programmming Paradigm for Soft Computing in Complex Systems", in Handbook of Nature-Inspired \& Innovative Computing, Ed: A.Zomaya, Springer Verlag, New York, 2006, pp.77-109.

[15] S.C.Manrubia et al., Emergence of dynamical order, World Scientific,Singapore ,2004..

[16] F.C.Moon, Chaotic and Fractal Dynamics, John Wiley, New York, 1999.

[17] M.E.J.Newman, "The structure and Function of complex Networks", Santa Fe Institute, see web.

[18] A. Pikovsky et al., Synchronization, Cambridge University Press, Cambridge, 2003.

[19] I.Prigogine,From being to becoming, W.H.Freeman and Co, San Francisco, 1980.

[20] M.R.Rose and G.V.Lauder, Adaptation, Academic Press, New York, 1996.

[21] F.Schweitzer, Brownian Agents and Particles, Springer Verlag, Berlin ,2002.

[22] G,D.Serugendo," Engineering Emergent Behaviour:A Vision", Lecture Notes in Artificial Intelligence,vol. 2927, Springer Verlag, New York, 2003 ,pp. 1-7

[23] R.C.Smith, Smart Material Systems,SIAM, Philadelphia, 2005.

[24] D. Strauss," On a general class of models for interaction", SIAM Review, vol.28,1986,pp.513-527..

[25] S.H.Strogatz,Sync:The emerging science of spontaneous Order, Hyperion Press, New York,2003

[26] S.Torquato,Random Heterogeneous Materials, Springer, New York, 2002

[27] D. Watts, Small Worlds, Princeton University Press, Princeton, 1999.

[28] S.Wolfram, A New kind of Science, Wolfram Media Inc., Champaign, Ill, 2002.

[29] M.Woolridge, Introduction to Multi-Agent systems, John Wiley, New York, 2002.

[30] M.Zak, M, et al., From Instability to Intelligence, Springer Verlag, New York ,1997.

[31] G.M.Zaslavsky,"Chaotic Dynamics and the origin of Statistical Laws”, Physics Today Vol.52(3), pp.39-45,1999. 